\title{
Expression of brain-derived neurotrophic factor in basolateral amygdala inputs to lateral septum is necessary for mice to identify socially novel individuals
}

Lionel A. Rodriguez ${ }^{\star 1,2}$, Sun-Hong Kim, $\mathrm{PhD}^{\star 2}$, Stephanie C. Page, PhD², Claudia V. Nguyen ${ }^{2}$, Elizabeth A. Pattie ${ }^{2}$, Henry L. Hallock, $\mathrm{PhD}^{2}$, Jessica Valerino ${ }^{2}$, Kristen R. Maynard, PhD², Andrew E. Jaffe ${ }^{1,2,3,4,5,6,7}$, Keri Martinowich, $\operatorname{PhD}^{1,2,4}$

1. Department of Neuroscience, Johns Hopkins School of Medicine, Baltimore, MD, 21205, USA

2. Lieber Institute for Brain Development, Johns Hopkins Medical Campus, Baltimore, MD, 21205, USA

3. Department of Genetic Medicine, McKusick-Nathans Institute of Genetic Medicine, Johns Hopkins University School of Medicine, Baltimore, MD, 21205, USA

4. Department of Psychiatry and Behavioral Sciences, Johns Hopkins School of Medicine, Baltimore, MD, 21205, USA

5. Center for Computational Biology, Johns Hopkins University, Baltimore, MD, 21205, USA

6. Department of Biostatistics, Johns Hopkins Bloomberg School of Public Health, Baltimore, MD, 21205, USA

7. Department of Mental Health, Johns Hopkins Bloomberg School of Public Health, Baltimore, MD, 21205, USA

${ }^{*}$ Equal Contribution

Correspondence:

Keri Martinowich

Lieber Institute for Brain Development

855 North Wolfe Street

Office \#382

Baltimore, MD, 21205.

keri.martinowich@libd.org

(410) 955-1510 


\section{ABSTRACT}

BACKGROUND: The lateral septum (LS) is activated by social novelty, and perturbing LS activity impairs social recognition. However, the neural circuits and cell signaling pathways that converge on the LS to mediate social behaviors aren't well understood. Multiple lines of evidence suggest that social behavior is influenced by brain-derived neurotrophic factor (BDNF) signaling through its receptor TrkB. However, whether BDNF-TrkB signaling mediates social behavior by affecting function in LS circuits is not known.

METHODS: We used single-molecule fluorescent in-situ hybridization to quantify expression of Ntrk2 (gene encoding TrkB) in LS. Viral transgenesis was used to induce cre-recombinase mediated TrkB knockdown in LS as well as for ablation and BDNF-depletion in neurons projecting from the basolateral amygdala (BLA) to the LS. We evaluated social recognition behavior using the three-chamber social interaction test, and assessed social novelty-induced cFos expression using fluorescence immunohistochemistry.

RESULTS: The majority of GABAergic neurons in LS express TrkB. TrkB knockdown in LS abolishes social novelty recognition, and decreases LS activation in response to social novelty. Ablating BLA-LS projection neurons abolishes social novelty recognition behavior, an effect that is phenocopied by selectively depleting BDNF in this circuit.

CONCLUSIONS: Social novelty recognition in the mouse requires both BDNF expression in BLA-LS projections neurons and intact TrkB signaling in the LS. These data support the hypothesis that BLA-LS projection neurons are a critical source of BDNF for activating LS TrkB signaling to control social novelty recognition.

Keywords: lateral septum, basolateral amygdala, BDNF, social novelty recognition, TrkB 


\section{INTRODUCTION}

Social deficits are prevalent in many psychiatric and neurodevelopmental disorders, including autism $(1,2)$ and schizophrenia (3). While behavioral manifestations of altered social behavior in these disorders are highly heterogeneous, deficits in attention to socially salient stimuli, and deficits in social recognition are well documented (3-5). Mice are a highly social species that display robust responses to novel conspecifics, such as attention to, and investigation of, novel individuals $(6,7)$. The three-chamber social approach task, which can be used to measure the amount of time a mouse spends in close proximity with another mouse, was developed and validated to assess sociability, social recognition, and social novelty preference (8). Although these behaviors can't fully encapsulate the complexities of human social behaviors, these rodent models can be used to study neurocircuitry that is relevant for social behavior impairments observed in human disorders.

The lateral septum (LS) is a predominantly GABAergic region in the basal forebrain that extends across a significant portion of the rostral-caudal axis and is subdivided into a dorsal, intermediate and ventral subregion (9). The LS is a potent modulator of social behaviors in both humans (10-13) and rodents $(9,14)$. Investigation of the LS in rodents demonstrated its involvement in social saliency $(15,16)$, social recognition (17-19), and social aggression (20, 21). The LS is also innervated by many regions involved in regulating various social behaviors, including the basolateral amygdala (BLA) (22), ventral hippocampus CA1 area (vCA1) (23), ventral tegmental area $(9,24)$, as well as the infralimbic and prelimbic cortices $(9,25,26)$. However, the inputs to the LS that mediate social recognition are not well understood, and the cell-signaling mechanisms in the LS that transmit this social information remain unclear.

Multiple lines of evidence suggest that the activity-dependent neurotrophin brain-derived neurotrophic factor (BDNF) is required for a variety of social behaviors (27-30). Signaling through its cognate receptor tropomyosin kinase $B$ (TrkB), BDNF regulates dendritic morphology, synapse formation and synaptic plasticity (31-33). Importantly, BDNF plays a critical role in supporting the function of GABAergic neurons (34-38), which rarely synthesize BDNF, but express TrkB $(39,40)$. GABAergic neurons rely on BDNF released from other cell types to activate TrkB receptor signaling, which is critical for their maturation and physiological function $(37,41-43)$. In line with its GABAergic composition, the LS is largely devoid of Bdnf transcript expression, but many neurons innervating the LS densely express BDNF $(44,45)$. Previous research has shown that catecholaminergic projections that express BDNF and innervate the LS influence the morphology and gene expression of a subpopulation of calbindin- 
expressing neurons in the LS (46). However, whether BDNF-TrkB signaling in the LS is important for the social behaviors mediated by the LS has not been investigated.

We hypothesized that 1) TrkB signaling in LS GABAergic neurons is important for regulating rodent social behavior, and that 2) neuronal projections from BDNF-rich limbic regions serve as a source of BDNF to activate TrkB signaling in LS to control this behavior. Key candidate regions included the BLA and VCA1 because both regions highly express BDNF (44), send projections to the $\operatorname{LS}(20,22,26)$, and have established roles in regulating social novelty $(9,16,47)$ and recognition $(23,48,49)$. Indeed, local knockdown of TrkB in the LS demonstrates a role for LS TrkB expression in social novelty and recognition behavior in mice. Moreover, we identify BLA-LS projections as critical for social novelty and recognition behavior in mice, and show that BDNF expression within these projection neurons is required for this behavior. In summary, the data demonstrate a vital role for LS TrkB signaling in social novelty recognition, and suggest that the BLA is a critical source of BDNF for activating LS TrkB receptors to control this behavior.

\section{METHODS AND MATERIALS}

\section{Animals}

Wild-type mice (C57BL/6J; stock \# 000664) and mice carrying a loxP-flanked Bdnf allele $\left(B d n f^{\mathrm{fm} 3 \mathrm{Jae}}\right.$; stock \# 004339, referenced in text as BDNF $\left.{ }^{\text {fl/fl}}\right)$ were purchased from The Jackson Laboratory. Mice carrying a loxP-flanked TrkB allele (strain $\mathrm{fB} / \mathrm{fB}$, referenced in text as TrkB ${ }^{\text {fl/fl }}$ $(50,51))$ were used for some experiments. TrkB ${ }^{f / f t}$ mice were maintained on a C57BL/6J background. Adult male mice were housed in a temperature-controlled environment with a 12:12 light/dark cycle and ad libitum access to food and water. All experimental animal procedures were approved by the Sobran Biosciences Institutional Animal Care and Use Committee.

\section{Behavior}

The three-chamber social interaction test arena consisted of three adjacent chambers separated by two clear plastic dividers, and connected by two open doorways. Mice were habituated for 3 consecutive days (10 min per day) prior to testing days. Mice were transferred to the testing room $1 \mathrm{~h}$ prior to testing. The test consisted of $5 \mathrm{~min}$ of habituation and two $10 \mathrm{~min}$ sessions (trial 1 and 2) with a 5 min inter-trial-interval. The subject mouse was kept in the center chamber during habituation. In the first 10 min session (trial 1), the subject mouse was allowed to freely investigate the three chambers. A novel mouse (stranger 1) was placed under an 
inverted metal cup in one of the side chambers, and another identical inverted empty cup was placed in the other side chamber. After the trial 1 session, the doorways were closed and the subject mouse was kept in the center chamber for $5 \mathrm{~min}$. In the second session (trial 2), another novel object mouse (stranger 2) was put in the empty cup and the subject mouse freely investigated the three chambers. Age-matched adult C57BL/6J male mice were used as object mice in this study, and housed under the same conditions as the subject mice. Each object novel/stranger mouse was used once a day in the three-chamber social interaction test. Time spent sniffing each cup was scored as interaction time manually in a blind manner using a Stopwatch+ program developed by the Center for Behavioral Neuroscience (cbn-atl.org) at Emory University $(52,53)$.

For full description of methods, see Supplementary information.

\section{RESULTS}

\section{Local knockdown of LS TrkB expression}

We used RNAscope single-molecule fluorescent in-situ hybridization to quantify expression of the gene encoding TrkB (Ntrk2 probe) in GABAergic neurons (Gad1 probe) across the rostral-caudal axis of the LS (Fig. 1A,B). Quantification of these data revealed that in wild-type mice, nearly all LS GABAergic neurons (85-90\%) expressed Ntrk2, a pattern that was consistent across the rostral-caudal axis (Fig. 1B). Next, we designed a genetic manipulation strategy to knockdown expression of TrkB in the LS. Specifically, we injected a cre recombinase-expressing virus (AAV5-EF1a:Cre) into the LS of mice carrying a floxed TrkB allele (TrkB ${ }^{f / f l}$ mice) to knockdown TrkB selectively in LS (Fig 1C). As controls, we generated mice that retained intact TrkB expression (TrkB ${ }^{f / f l}$ mice injected into the LS with a non-Cre expressing virus; AAV5-EF1a:EYFP) (Fig. 1C). Four weeks following injection of the viruses, we micro-dissected the LS and frontal cortex from each mouse, and quantified protein expression of both full-length and truncated TrkB in individual mice (Fig. 1D-F). Expression of cre recombinase in TrkB $^{\text {fl/fl }}$ mice resulted in $\sim 86 \%$ knockdown of full-length TrkB expression selectively in the LS, but not in the frontal cortex (Fig. 1D,E). Based on predicted sequence translation, removal of the floxed portion of the $N$ trk2 gene in $\operatorname{TrkB}^{f / f l}$ mice is not expected to affect expression of the truncated TrkB isoform (51), and hence it was unsurprising that expression of truncated TrkB remained unchanged across experimental groups (Fig. 1D,F).

\section{Knockdown of LS TrkB abolishes social novelty discrimination}


We next used this local knockdown strategy to investigate the necessity of TrkB signaling in social novelty and recognition using the three chamber social interaction task (Fig $2 A, B)$. This task uses a three-chambered box to quantify the time a mouse spends examining socially novel individuals. In trial 1 , the mouse is exposed to a novel mouse (stranger mouse 1) in one of the outer chambers while the other outer chamber remains empty. In trial 2, stranger mouse 1 remains in the same chamber (now the familiar mouse) while a new mouse (stranger mouse 2) is placed in the other chamber.

Control mice with intact TrkB expression showed expected social novelty behavior, spending more time investigating the novel mouse in trial 1 and trial 2 (Fig. 2C). However, while mice with LS TrkB knockdown showed expected social novelty behavior in trial 1, these mice spent equal time with the familiar and novel mice in trial 2 (Fig. 2D). To evaluate differences in the ability of control and LS TrkB knockdown mice to discriminate between individuals we generated a discrimination index for trial 2 , which is a percentage of the time spent with the novel individual minus the time spent with the familiar individual over the total trial time. The decreased discrimination index in LS TrkB knockdown mice suggests that loss of intact TrkB may impact the ability to discriminate between individual mice (Fig. 2E).

To rule out that the impairment in attention to social novelty discrimination was due to olfactory impairment, both controls and mice with LS TrkB knockdown were subjected to a four trial odor discrimination task (Fig. S1A). LS TrkB knockdown did not impair odor discrimination (Fig. S1B). We also assessed the necessity of TrkB signaling in LS for a number of fear- and anxiety-related behaviors that are known to engage LS circuitry. LS TrkB knockdown did not alter behavior in the elevated plus maze (Fig. S1C), nor did it impact the ability of LS TrkB knockdown mice to acquire, retrieve or extinguish fear memories (Fig. S1D). These data support the hypothesis that the deficits observed following knockdown of LS TrkB are specific to social behavior.

\section{LS TrkB knockdown abolishes socially induced c-Fos Expression}

To map neural activity patterns in LS cells following exposure to novel social stimuli, we performed fluorescent immunohistochemistry for c-Fos across the rostral-caudal divide of the LS (Fig. 3A) in wild-type mice exposed to a social stimulus (a novel male mouse) compared to a non-social stimulus (a novel object, conical tube)(Fig. 3B). c-Fos quantification was performed using the open-source biological imaging software Fiji (for detailed description of methods, see Supplementary information and Fig S2A). In the middle portion of the LS, significantly more cells expressed c-Fos in response to social stimuli compared to the non-social stimulus (Fig. 3C). 
This effect mapped most strongly to the intermediate and ventral subregions of the LS (Fig. 3D). The number of c-Fos expressing cells in all other subregions within the rostral (Fig. S2B) and caudal (Fig. S2C) portions of the LS remained relatively consistent across the social and nonsocial conditions suggesting that these effects on cell recruitment in the LS are restricted to the middle portion of the LS.

To investigate whether TrkB signaling contributed to LS-responsivity to socially novel stimuli, we generated an additional cohort of LS TrkB knockdown and control mice using the same viral strategy described above (Fig. 3E). Compared to controls, we detected significantly fewer cells expressing c-Fos in response to novel social stimuli in the middle portion of the LS in LS TrkB knockdown mice (Fig. 3F). All subregions (dorsal, intermediate, and ventral) within the middle portion of the LS displayed blunted c-Fos expression in response to a socially novel individual (Fig. 3G). However, LS TrkB knockdown had no effect on c-Fos expression induced in response to socially novel individuals within the rostral (Fig. S3B) and caudal (Fig. S3C) portions of the LS compared to controls.

\section{BLA projections, but not vCA1 projections, to the LS are necessary for social novelty discrimination}

While the LS is critical for controlling responses to social novelty (16-19), the brain regions that transmit information to the LS for proper execution of social novelty discrimination have not been identified. We noted the BLA and the vCA1 as candidate regions because both have established roles in regulating social novelty $(9,16,47)$ and recognition $(23,48,49)$, and send projections to the $\operatorname{LS}(22,26)$. To determine the importance of projections from the BLA or vCA1 to the LS in controlling social novelty behavior, we used a diphtheria toxin A (DtA)mediated viral circuit elimination strategy. Specifically, we injected two viruses with retrograde tropism (AAVrg-CB7.CI:EGFP and AAVrg-EF1a:mCherry-IRES-Cre) into the LS bilaterally, causing expression of eGFP, mCherry, and Cre in cells projecting to LS (Fig. 4A). In our regions of interest (separate cohorts for BLA and vCA1) we also injected a virus (AAV5-EF1a:mCherryFLEX-dtA) expressing mCherry and Cre-dependent DtA (Fig. 4A). In this scenario, cell bodies in the BLA or VCA1 that also sent projections to the LS expressed Cre-recombinase, and hence Cre-mediated expression of DtA in the BLA-LS or VCA1-LS neuronal projections. This manipulation caused elimination of the eGFP signal in those ablated projections, but retention of the mCherry signal in non-projecting cells (Fig. 4B). Control mice with an intact BLA-LS circuit showed expected social novelty behavior, spending more time investigating the novel mouse in trial 1 and trial 2 in the three chamber social recognition task (Fig 4C). Mice with ablated BLA- 
LS projections displayed expected social novelty behavior in trial 1, but in trial 2 these mice spent equal time with the familiar and novel mice (Fig. 4D). In addition, mice with intact BLA-LS projection neurons discriminated better than mice with ablated BLA-LS projection neurons according to their relative percentage of time spent with the novel versus familiar mouse in trial 2 (Fig. 4E). Both control mice with an intact vCA1-LS circuit (Fig. 4F) and a vCA1-LS ablated circuit (Fig. 4G) showed the expected social novelty behavior in trial 1 and trial 2. Neither group differed in their ability to discriminate between the novel and familiar mice in trial 2 (Fig. $4 \mathrm{H}$ ). Our ablation studies demonstrate a role in BLA-LS neurons, and not vCA1-LS neurons, in regulating social novelty discrimination.

\section{Expression of BDNF within BLA projections to the LS is necessary for social novelty discrimination}

Neurons in the BLA strongly express BDNF (44), and the BLA sends projections to the LS (22). Hence, BLA-LS neurons are a strong candidate source for providing BDNF to LS TrkBexpressing neurons that regulate social novelty. To assess whether BDNF expression is necessary in BLA-LS projections to control social novelty behavior, we used a viral strategy combining the Flp recombinase (FlpO) and cre recombinase systems in mice carrying a floxed Bdnf allele $\left(\mathrm{BDNF}^{f / f}\right)$. This strategy allowed us to eliminate BDNF selectively from BLA-LS projections (Fig. 5A). We injected a retrograde virus in the LS (AAVrg-EF1a:Flpo) of BDNF fl/fl mice, causing FlpO expression in all neuronal inputs to the LS. We simultaneously injected a virus (AAV8-EF1a:fDIO-mCherry-P2A-Cre) expressing Flp-dependent mCherry and crerecombinase in the BLA. Control mice were injected with a virus (AAV8-EF1a:fDIO-mCherry) into the BLA that expressed Flp-dependent mCherry. In the experimental group, BLA neurons projecting to the LS express cre-recombinase in a FlpO-dependent manner. Expression of cre leads to excision of the floxed BDNF allele and Cre-dependent expression of mCherry (Fig. 5B). Control mice with intact BDNF expression in the BLA-LS circuit showed expected social novelty behavior, spending more time investigating the novel mouse in trial 1 and trial 2 in the three chamber social recognition task (Fig. 5C). Mice with knockdown of BDNF expression in BLA-LS projection neurons showed expected social novelty behavior in trial 1, but in trial 2 these mice spent equal time with the familiar and novel mice (Fig. 5D). Moreover, mice with decreased BDNF expression in BLA-LS projection neurons display poorer discrimination between individuals compared to animals with intact BDNF expression in BLA-LS projection neurons according to their relative percentage of time spent with the novel versus familiar 
mouse in trial 2 (Fig. 5E). In summary, BDNF expression in BLA-LS neurons is critical for social novelty discrimination.

\section{DISCUSSION}

\section{TrkB signaling in the LS critically regulates social novelty behavior}

Our findings reveal a novel role for TrkB signaling in the LS in controlling social novelty behavior in mice. The LS is involved in controlling social salience $(15,16)$ and social recognition (17-19), but little is known about the cellular and molecular mechanisms by which social information is relayed to the LS. We found that knocking down LS TrkB abolished social novelty behavior in the three-chamber social interaction task, suggesting that TrkB expression in the LS is necessary for discriminating socially novel conspecifics from those that are socially familiar. Importantly, the effects of TrkB knockdown did not impair olfactory discrimination or fear learning, highlighting the specificity of LS TrkB signaling in controlling social novelty behavior. The LS is spatially complex, containing dorsal, ventral, and intermediate subregions that span its entire rostral-caudal axis. Importantly, these subregions have functional distinctions, for example the dorsal and ventral LS control different aspects of social aggression in both males (23) and females (54). While elevated c-Fos expression in the LS in response to social stimuli is documented $(15,16)$, how this activity maps to the spatial topography of the LS has not been rigorously explored. Using c-Fos neural activity mapping, we demonstrated that the intermediate and ventral subregions of the middle portion of the LS are the socially responsive loci. Previous results suggested that social novelty-induced activity in the LS was concentrated more rostrally than caudally (15), which is consistent with our results. In addition, we demonstrated that TrkB expression in the LS is necessary for LS responsiveness to social stimuli. Specifically, recruitment of neural activity in the intermediate and ventral subregions of the middle portion of the LS in response to social stimuli is significantly blunted by LS TrkB knockdown. While our data suggests that TrkB expression in the LS gates the region's response to social stimuli, further work with acute manipulations of TrkB receptors in vivo could reveal the fast acting effects of BDNF-TrkB signaling within the LS in controlling social behaviors. This is important, since the chronic effects of TrkB knockdown in LS in the model utilized here could impact the physiological function of TrkB-expressing inhibitory neurons, which could impact the strength of inhibitory synapses. This in turn could impact connectivity within the LS, or inputs to the LS, contributing to the impairments in social behaviors that were observed. Hence, to better define the kinetics and mechanisms by which LS TrkB signaling controls social discrimination, a more 
transient approach to blocking TrkB activation (e.g. using TrkB antagonists) could be pursued in follow up studies.

\section{BLA projections to the LS control social novelty behavior}

Our data reveal a novel role for direct neuronal projections from the BLA to the LS in controlling social novelty discrimination. The BLA is responsive to socially novel conspecifics $(16,47)$, and controls social discrimination (49), but the brain regions with which the BLA communicates to regulate these behaviors are not well established. We found that ablation of neuronal projections from the BLA to the LS abolishes social novelty behavior, suggesting that this circuit is necessary for discriminating socially novel conspecifics from those that are socially familiar. The BLA is critical for valence processing in a variety of contexts $(55,56)$, which may extend to processing social stimuli (57). The BLA receives inputs from multiple regions that can transmit social information, including the olfactory systems of the brain (58). Further work identifying the extended circuits that relay social information to the BLA may help clarify the role of the BLA in controlling social novelty and recognition.

Importantly, ablating projections from vCA1 to the LS did not alter social discrimination behavior. This finding is somewhat surprising as vCA1 has been implicated in controlling responses to social novelty (16) and regulating social memories $(23,48)$, and LS projections from adjacent subregions of the hippocampus, including dorsal CA2 (dCA2), are critical for controlling social aggression (20). While dCA2 projections to vCA1 are critical for social recognition (23), this social information may not be transmitted from ventral hippocampal circuitry to the LS to control social novelty. Further research may determine if other portions of the ventral hippocampus, such as $\mathrm{dCA} 2$, regulate social novelty and recognition, in addition to aggression. Interestingly, activation versus inhibition of BLA projections to the ventral hippocampus bidirectionally controls time spent socializing (57), which may point to the BLA as a central transmitter of social information to limbic circuitry.

\section{BLA-derived BDNF signaling in the LS is critical for social novelty behavior}

While our data identify BLA-LS projection neurons as critical for regulating social novelty and recognition behavior, the molecular mechanisms by which these neurons influence signaling in the LS to control this behavior is unknown. Our data demonstrate that TrkB expression in the LS is critical for social novelty and recognition behavior (Fig1D-G), but the source of BDNF for activating LS TrkB receptors is unclear. BDNF is not synthesized within the LS (44), but is highly expressed in BLA neurons (44), and given our data showing that BLA-LS 
projection neurons are also critical for this behavior, we reasoned that BDNF produced in BLALS projection neurons could be a critical source for activating LS TrkB receptors to control this behavior. Indeed, when we knocked down BDNF expression selectively in BLA-LS projection neurons, we found that this manipulation phenocopied the behavioral deficits caused by local TrkB knockdown in the LS.

$B d n f$ transcription is controlled by nine different unique promoters, each of which drives expression of a short, non-coding exon that is spliced to a single, common coding exon that produces the mature BDNF protein $(27,59,60)$. Disruption of BDNF production from specific Bdnf promoters impacts a variety of behaviors, including social aggression $(27,60)$ and maternal social behaviors (27). For example, our group recently demonstrated that BDNF production from promoters I and II controls maternal behaviors by influencing BDNF-dependent cellular functions in the hypothalamus (27). While BDNF-TrkB signaling in the LS is critical for regulating social novelty and recognition, it remains to be understood which promoters drive Bdnf expression in the BLA. Future experiments that determine this information may provide further insight into specific cellular and molecular mechanisms, and therefore specific stimuli, that facilitate the ability of BLA circuitry to control these social behaviors.

Similar to the rodent BLA, the non-human primate BLA (61) and the human BLA (62-64) are implicated in controlling a variety of social behaviors. Better understanding the nature of limbic control over social behaviors, such as social saliency and social memory, which are frequently disrupted in neuropsychiatric and neurodevelopmental disorders, is important to understand the etiology of these disorders, and develop targeted treatments for these symptoms. While the cellular and molecular mechanisms that control these behaviors are complex, given the prominent role of BDNF-TrkB signaling in controlling many facets of synaptic function, it is likely that this pathway contributes to regulating a variety of social behaviors. Supporting this notion, rare genetic disorders that cause BDNF haploinsufficiency as well as BDNF gene variants in humans are linked with aggression as well as other aspects of social functioning (65-67). In addition to BDNF, a recent genome-wide association study of anxiety, which included a large population of human subjects with social anxiety, identified a single nucleotide polymorphism in NTRK2, the gene encoding the TrkB receptor as significantly contributing to genetic risk (68). Better understanding the cellular and molecular mechanisms by which BDNF-TrkB signaling exerts control over social behaviors may help us further elucidate the circuit and molecular mechanisms that are relevant to the manifestation of both normal and abnormal social behavior. 


\section{Ethological Considerations}

A limitation of our studies is that they were conducted exclusively in male mice. While male and female mice show many overlaps in social behaviors, there are various documented differences, including how they establish social hierarchies $(69,70)$, neural coding of social behaviors (71), and certain aspects of social recognition (72). Hence, direct comparisons of males and females in social novelty and recognition paradigms may be difficult to interpret at the behavioral level. Despite these sex differences in social behavior, there are several reasons why it is important for future studies to examine a potential role for BDNF-TrkB signaling in BLALS circuitry in the context of sex-specific social behaviors. First, prevalence rates (73), as well as genetic risk for disorders that often feature social deficits are not equal across sex and gender in humans (74-78). Second, BDNF-TrkB signaling and expression is significantly impacted by sex and hormones (79-81).

Conducting these studies, however, will require careful experimental design that takes into consideration the ethological aspects of female social behaviors. Specifically, the presence of a socially novel individual can signal different information to a male versus a female mouse, and can vary by context. For example, female mice establish different social hierarchies depending on the presence of male mice (70). This hierarchy leads to territoriality and aggression in some females toward males and females, while the non-aggressive females typically were breeders (70), which is remarkable given that female aggression is usually only displayed by postpartum dams in defense of their pups (82). The lack of male mice leads to little to no aggression in female mice. Male mice, on the other hand, typically display territoriality and aggression in male-male interactions $(70,83)$, but seldom display these behaviors in malefemale interactions (70). It is also noteworthy that this female hierarchy and aggressive social behavior is found in instances where mice are housed in larger chambers with more individuals than the typical 4-6 mice per cage housing scheme used in most academic vivariums (70).

Despite the difficulties in making direct comparisons between male and female mice in studies of social behaviors, future studies can implement well designed experiments to study sex-specific differences in social novelty and recognition. For example, studying female social novelty in postpartum mice or dominant female mice raised in complex social structures will elucidate whether the circuits that enables normal male responses to social novelty are also necessary for controlling female social novelty. However, even then, this beckons the question about whether these female-male (or female-female) and male-male responses to social novelty are parallel at an ethological level. 


\section{Conclusions}

Collectively, the presented data demonstrate a critical role for TrkB signaling in LS neurons in controlling social novelty recognition, and suggest that the BLA is a critical source of BDNF in activating these TrkB receptors to control this behavior. The data provide novel information about the broader role of signaling in amygdala-septal circuits, and provide a foundation for future studies to better delineate how molecular and cellular signaling in these circuits impacts social functioning.

\section{FIGURE LEGENDS}

Figure 1. Tropomyosin receptor kinase $B$ (TrkB) is highly expressed in the lateral septum (LS) and can be effectively depleted virally. (A) Illustration depicting the anatomical boundaries across the rostral-caudal axis of the LS. (B) Fluorescent in-situ hybridization in sections of mouse LS $(n=4)$ demonstrates Gad1-positive cells highly express Ntrk2 throughout the rostralcaudal divisions. (C) Viral strategy using Cre-induced TrkB knockdown in the LS of TrkB ${ }^{\mathrm{fl} / \mathrm{fl}}$ mice to quantify extent of TrkB knockdown quantification. (D) Western blot images for full-length TrkB (F-TrkB), truncated TrkB (T-TrkB), and a-Tubulin (aTub). (E) Relative expression levels of FTrkB protein were significantly lower in the LS of the experimental group (AAV-Cre in LS of TrkB $^{f / / f l}$ mice, $\left.n=5\right)$ compared to the control group (AAV-eGFP in LS of TrkB ${ }^{f / f l}$ mice, $\left.n=6\right)\left(t_{9}=\right.$ 5.015, $p=0.0014)$. (F) Relative expression levels for T-TrkB protein were similar between experimental and control mice.

Figure 2. Tropomyosin receptor kinase $B$ (TrkB) expression in the lateral septum (LS) is required for social novelty recognition behavior in mice. (A) Description of three chamber social interaction experiment. (B) Viral strategy using Cre-induced knockdown of TrkB expression in the $L S$ of TrkB $^{\mathrm{fl} / \mathrm{fl}}$ mice prior to social novelty recognition. (C) Mice with intact TrkB expression in the LS $(n=7)$ display intact social novelty recognition behavior. In both trial $1\left(t_{6}=10.99, p<\right.$ $0.0001)$ and trial $2\left(t_{6}=5.921, p=0.001\right)$ control mice spend more time investigating the socially novel mouse. (D) TrkB knockdown in LS $(n=11)$ abolishes the social novelty phenotype observed in trial $2\left(t_{10}=1.898, p=0.088\right)$, but not trial $1\left(t_{10}=5.615, p=0.0002\right)$. (E) Mice with intact TrkB expression in the LS show better social discrimination between the socially novel and familiar mice in Trial 2 when compared to mice with TrkB knockdown in the $L S\left(U_{11,7}=12, p\right.$ $=0.0154)$. 
Figure 3. Tropomyosin receptor kinaseB (TrkB) expression in the lateral septum (LS) is required for social novelty induced c-Fos expression. (A) Illustration depicting the anatomical boundaries across the rostral-caudal axis of the LS, denoting the dorsal (d), intermediate (i), and ventral (v) subregions of the LS. (B) Description of social or non-social cues used to induce cFos expression. (C) Induction of c-Fos is significantly increased by social cues in the middle portion of the LS $\left(t_{6}=5.543, p=0.004\right)$, (D) an effect seen specifically in the intermediate $\left(t_{6}=\right.$ $3.697, p=0.030)$ and ventral subdivisions $\left(t_{6}=3.131, p=0.040\right)$ of the middle LS. (E) Viral strategy using Cre-induced TrkB knockdown in the LS of TrkB ${ }^{f / / f l}$ mice prior to examining social interaction induced c-Fos expression. (F) Knockdown of TrkB in the LS significantly decreases cell recruitment in the middle portion of the LS following social stimuli $\left(t_{9}=3.069, p=0.0396\right)$, (G) an effect seen across the dorsal $\left(t_{9}=3.049, p=0.029\right)$, intermediate $\left(t_{9}=3.254, p=0.029\right)$, and ventral $\left(t_{9}=2.342, p=0.044\right)$ subdivisions of the middle $L S$.

Figure 4. Ablating inputs to the lateral septum (LS) from basolateral amygdala (BLA), but not ventral CA1 (vCA1), abolishes social novelty recognition behavior in mice. (A) Cartoon of viral strategy used to selectively eliminate inputs to the LS from either the BLA or vCA1 utilizing retrograde labeling and Cre-mediated expression of diphtheria toxin A (DtA) (left panel). Schematics depict ablation in cohort where BLA-LS projections are targeted (middle panel), and ablation in cohort where vCA1-LS projections are targeted. (B) Representative images show that in the cohort where DtA is expressed in BLA (top row), BLA-LS projections are ablated, while vCA1-LS projections are intact, while in the cohort where DtA is expressed in vCA1 (bottom row), vCA1-LS projections are ablated while BLA-LS projections remain intact . (C) Mice with the BLA-LS circuit intact $(n=10)$ display normal social novelty recognition behavior. In both trial $1\left(t_{9}=7.808, p<0.0001\right)$ and trial $2\left(t_{9}=5.17, p=0.0006\right)$ they spend more time investigating the socially novel mouse. (D) Ablation of the BLA-LS circuit $(n=10)$ abolishes the social novelty phenotype observed in trial $2\left(t_{9}=0.6048, p=0.56\right)$, but not trial $1\left(t_{9}=12.74, p<\right.$ 0.0001). (E) Mice with an intact BLA-LS circuit show better social discrimination between the socially novel and familiar mice in Trial 2 than mice with an ablated BLA-LS circuit $\left(\cup_{10,10}=17, p\right.$ $=0.0115)$. (F) Mice with the vCA1-LS circuit intact $(n=8)$ display normal social novelty recognition behavior. In both trial $1\left(t_{7}=8.449, p<0.0001\right)$ and trial $2\left(t_{7}=10.5, p<0.0001\right)$ they spend more time investigating the socially novel mouse. $(G)$ Ablation of the vCA1-LS circuit $(n=8)$ does not alter social novelty behavior in trial $1\left(t_{7}=5.235, p=0.0012\right)$ or trial $2\left(t_{7}=2.979\right.$, $p=0.0206)$. $(H)$ Mice with an intact vCA1-LS circuit and mice with an ablated vCA1-LS circuit show no difference in social discrimination $\left(U_{9,9}=30, p=0.8527\right)$. 
Figure 5. Knockdown of brain-derived neurotrophic factor (BDNF) in basolateral amygdala (BLA) inputs to the lateral septum (LS) abolishes social novelty recognition behavior in mice. (A) Schematic of viral strategy used to deplete BDNF expression in BLA-LS projections in BDNF(f/fl mice. (B) Representative images of viral expression of mCherry within the BLA. (C) Mice with intact BDNF expression in BLA-LS projections $(n=9)$ display normal social novelty recognition behavior. In both trial $1\left(t_{8}=5.73, p=0.0004\right)$ and trial $2\left(t_{8}=6.842, p=0.0001\right)$ they spend more time investigating the socially novel mouse. (D) BDNF depletion in BLA-LS projections ( $n=9$ mice) abolishes the social novelty phenotype observed in trial 2 ( $t_{8}=1.763, p=0.116$ ), but not trial $1\left(t_{8}=5.456, p=0.0006\right)$. ( $\left.E\right)$ Mice with intact BDNF expression in BLA-LS projections display better social discrimination between the socially novel and familiar mice in trial 2 than mice with BDNF knockdown in BLA-LS projections $\left(U_{9,9}=9, p=0.004\right)$.

\section{ACKNOWLEDGEMENTS}

This work was supported by internal funding from the Lieber Institute for Brain Development, and the National Institute of Mental Health (R01MH105592 to KM). Andrew E. Jaffe is now a full time employee at Neumora Therapeutics, a for-profit biotechnology company, which is unrelated to the contents of this manuscript. Sun-Hong Kim is now employed by Genentech. Their contributions to the manuscript were made while previously employed by the Lieber Institute for Brain Development. No other authors have financial relationships with commercial interests, and the authors declare no competing interests. We thank members of the Martinowich and Maynard laboratories for helpful comments and suggestions.

\section{REFERENCES}

1. Chevallier C, Kohls G, Troiani V, Brodkin ES, Schultz RT (2012): The social motivation theory of autism. Trends Cogn Sci (Regul Ed). 16(4): 231-39.

2. Happé F, Ronald A (2008): The "fractionable autism triad": a review of evidence from behavioural, genetic, cognitive and neural research. Neuropsychol. Rev. 18(4): 287-304.

3. Marwick K, Hall J (2008): Social cognition in schizophrenia: a review of face processing. Br. Med. Bull. 88(1): 43-58.

4. Mier D, Kirsch P (2017): Social-cognitive deficits in schizophrenia. Curr. Top. Behav. 
Neurosci. 30: 397-409.

5. Weigelt S, Koldewyn K, Kanwisher N (2012): Face identity recognition in autism spectrum disorders: a review of behavioral studies. Neurosci. Biobehav. Rev. 36(3): 1060-84.

6. Pobbe RLH, Pearson BL, Defensor EB, Bolivar VJ, Blanchard DC, Blanchard RJ (2010): Expression of social behaviors of $c 57 \mathrm{bl} / 6 \mathrm{j}$ versus btbr inbred mouse strains in the visible burrow system. Behav. Brain Res. 214(2): 443-49.

7. Moy SS, Nadler JJ, Perez A, Barbaro RP, Johns JM, Magnuson TR, et al. (2004): Sociability and preference for social novelty in five inbred strains: an approach to assess autistic-like behavior in mice. Genes Brain Behav. 3(5): 287-302.

8. Lo S-C, Scearce-Levie K, Sheng M (2016): Characterization of social behaviors in caspase-3 deficient mice. Sci. Rep. 6: 18335.

9. Sheehan TP, Chambers RA, Russell DS (2004): Regulation of affect by the lateral septum: implications for neuropsychiatry. Brain Res. Brain Res. Rev. 46(1): 71-117.

10. White SF, Brislin S, Sinclair S, Fowler KA, Pope K, Blair RJR (2013): The relationship between large cavum septum pellucidum and antisocial behavior, callous-unemotional traits and psychopathy in adolescents. J. Child Psychol. Psychiatry. 54(5): 575-81.

11. Raine A, Lee L, Yang Y, Colletti P (2010): Neurodevelopmental marker for limbic maldevelopment in antisocial personality disorder and psychopathy. Br. J. Psychiatry. 197(3): 186-92.

12. Crooks D, Anderson NE, Widdows M, Petseva N, Koenigs M, Pluto C, et al. (2018): The relationship between cavum septum pellucidum and psychopathic traits in a large forensic sample. Neuropsychologia. 112: 95-104.

13. Sarwar M (1989): The septum pellucidum: normal and abnormal. AJNR Am J Neuroradiol. 10(5): 989-1005.

14. Sheehan T, Numan M (2000): The septal region and social behavior. In: Numan R, editor. The behavioral neuroscience of the septal region, New York, NY: Springer New York, pp. 
175-209.

15. Shin S, Pribiag H, Lilascharoen V, Knowland D, Wang X-Y, Lim BK (2018): Drd3 signaling in the lateral septum mediates early life stress-induced social dysfunction. Neuron. 97(1): 195-208.e6.

16. Borelli KG, Blanchard DC, Javier LK, Defensor EB, Brandão ML, Blanchard RJ (2009): Neural correlates of scent marking behavior in c57bl/6j mice: detection and recognition of a social stimulus. Neuroscience. 162(4): 914-23.

17. Unable to find information for 896535.

18. Lukas M, Bredewold R, Landgraf R, Neumann ID, Veenema AH (2011): Early life stress impairs social recognition due to a blunted response of vasopressin release within the septum of adult male rats. Psychoneuroendocrinology. 36(6): 843-53.

19. Everts HG, Koolhaas JM (1999): Differential modulation of lateral septal vasopressin receptor blockade in spatial learning, social recognition, and anxiety-related behaviors in rats. Behav. Brain Res. 99(1): 7-16.

20. Leroy F, Park J, Asok A, Brann DH, Meira T, Boyle LM, et al. (2018): A circuit from hippocampal ca2 to lateral septum disinhibits social aggression. Nature. 564(7735): 21318.

21. Wong LC, Wang L, D’Amour JA, Yumita T, Chen G, Yamaguchi T, et al. (2016): Effective modulation of male aggression through lateral septum to medial hypothalamus projection. Curr. Biol. 26(5): 593-604.

22. Hintiryan H, Bowman I, Johnson DL, Korobkova L, Zhu M, Khanjani N, et al. (2021): Connectivity characterization of the mouse basolateral amygdalar complex. Nat. Commun. 12(1): 2859.

23. Meira T, Leroy F, Buss EW, Oliva A, Park J, Siegelbaum SA (2018): A hippocampal circuit linking dorsal ca2 to ventral ca1 critical for social memory dynamics. Nat. Commun. 9(1): 4163. 
24. Reddy IA, Pino JA, Weikop P, Osses N, Sørensen G, Bering T, et al. (2016): Glucagonlike peptide 1 receptor activation regulates cocaine actions and dopamine homeostasis in the lateral septum by decreasing arachidonic acid levels. Transl. Psychiatry. 6: e809.

25. Matthews GA, Tye KM (2019): Neural mechanisms of social homeostasis. Ann. N. Y. Acad. Sci. 1457(1): 5-25.

26. Risold PY, Swanson LW (1997): Connections of the rat lateral septal complex 1published on the world wide web on 2 june 1997. 1. Brain Res. Rev. 24(2-3): 115-95.

27. Maynard KR, Hobbs JW, Phan BN, Gupta A, Rajpurohit S, Williams C, et al. (2018): BDNF-trkb signaling in oxytocin neurons contributes to maternal behavior. eLife. 7:

28. Unable to find information for 3606217 .

29. Berton O, McClung CA, Dileone RJ, Krishnan V, Renthal W, Russo SJ, et al. (2006): Essential role of bdnf in the mesolimbic dopamine pathway in social defeat stress. Science. 311(5762): 864-68.

30. Ito W, Chehab M, Thakur S, Li J, Morozov A (2011): BDNF-restricted knockout mice as an animal model for aggression. Genes Brain Behav. 10(3): 365-74.

31. Huang EJ, Reichardt LF (2001): Neurotrophins: roles in neuronal development and function. Annu. Rev. Neurosci. 24: 677-736.

32. Chao MV (2003): Neurotrophins and their receptors: a convergence point for many signalling pathways. Nat. Rev. Neurosci. 4(4): 299-309.

33. Song M, Martinowich K, Lee FS (2017): BDNF at the synapse: why location matters. Mol. Psychiatry. 22(10): 1370-75.

34. Rutherford LC, DeWan A, Lauer HM, Turrigiano GG (1997): Brain-derived neurotrophic factor mediates the activity-dependent regulation of inhibition in neocortical cultures. $J$. Neurosci. 17(12): 4527-35.

35. Marty S, Berzaghi MdaP, Berninger B (1997): Neurotrophins and activity-dependent plasticity of cortical interneurons. Trends Neurosci. 20(5): 198-202. 
36. Woo NH, Lu B (2006): Regulation of cortical interneurons by neurotrophins: from development to cognitive disorders. Neuroscientist. 12(1): 43-56.

37. Hill JL, Jimenez DV, Mai Y, Ren M, Hallock HL, Maynard KR, et al. (2019): Cortistatinexpressing interneurons require trkb signaling to suppress neural hyper-excitability. Brain Struct. Funct. 224(1): 471-83.

38. Maynard KR, Kardian A, Hill JL, Mai Y, Barry B, Hallock HL, et al. (2020): TrkB signaling influences gene expression in cortistatin-expressing interneurons. eNeuro. 7(1):

39. Cellerino A, Maffei L, Domenici L (1996): The distribution of brain-derived neurotrophic factor and its receptor trkb in parvalbumin-containing neurons of the rat visual cortex. Eur. J. Neurosci. 8(6): 1190-97.

40. Gorba T, Wahle P (1999): Expression of trkb and trkc but not bdnf mrna in neurochemically identified interneurons in rat visual cortex in vivo and in organotypic cultures. Eur. J. Neurosci. 11(4): 1179-90.

41. Itami C, Kimura F, Nakamura S (2007): Brain-derived neurotrophic factor regulates the maturation of layer 4 fast-spiking cells after the second postnatal week in the developing barrel cortex. J. Neurosci. 27(9): 2241-52.

42. Huang ZJ, Kirkwood A, Pizzorusso T, Porciatti V, Morales B, Bear MF, et al. (1999): BDNF regulates the maturation of inhibition and the critical period of plasticity in mouse visual cortex. Cell. 98(6): 739-55.

43. Zheng K, An JJ, Yang F, Xu W, Xu Z-QD, Wu J, et al. (2011): TrkB signaling in parvalbumin-positive interneurons is critical for gamma-band network synchronization in hippocampus. Proc Natl Acad Sci USA. 108(41): 17201-6.

44. Conner JM, Lauterborn JC, Yan Q, Gall CM, Varon S (1997): Distribution of brain-derived neurotrophic factor (bdnf) protein and mrna in the normal adult rat cns: evidence for anterograde axonal transport. J. Neurosci. 17(7): 2295-2313.

45. Luo F, Mu Y, Gao C, Xiao Y, Zhou Q, Yang Y, et al. (2019): Whole-brain patterns of the 
presynaptic inputs and axonal projections of bdnf neurons in the paraventricular nucleus. J. Genet. Genomics. 46(1): 31-40.

46. Fawcett JP, Alonso-Vanegas MA, Morris SJ, Miller FD, Sadikot AF, Murphy RA (2000): Evidence that brain-derived neurotrophic factor from presynaptic nerve terminals regulates the phenotype of calbindin-containing neurons in the lateral septum. J. Neurosci. 20(1): 274-82.

47. Ferri SL, Kreibich AS, Torre M, Piccoli CT, Dow H, Pallathra AA, et al. (2016): Activation of basolateral amygdala in juvenile c57bl/6j mice during social approach behavior. Neuroscience. 335: 184-94.

48. Deng X, Gu L, Sui N, Guo J, Liang J (2019): Parvalbumin interneuron in the ventral hippocampus functions as a discriminator in social memory. Proc Natl Acad Sci USA. 116(33): 16583-92.

49. Garrido Zinn C, Clairis N, Silva Cavalcante LE, Furini CRG, de Carvalho Myskiw J, Izquierdo I (2016): Major neurotransmitter systems in dorsal hippocampus and basolateral amygdala control social recognition memory. Proc Natl Acad Sci USA. 113(33): E4914-9.

50. Grishanin RN, Yang H, Liu X, Donohue-Rolfe K, Nune GC, Zang K, et al. (2008): Retinal trkb receptors regulate neural development in the inner, but not outer, retina. Mol. Cell. Neurosci. 38(3): 431-43.

51. Baydyuk M, Russell T, Liao G-Y, Zang K, An JJ, Reichardt LF, et al. (2011): TrkB receptor controls striatal formation by regulating the number of newborn striatal neurons. Proc Natl Acad Sci USA. 108(4): 1669-74.

52. Burkett JP, Andari E, Johnson ZV, Curry DC, de Waal FBM, Young LJ (2016): Oxytocindependent consolation behavior in rodents. Science. 351(6271): 375-78.

53. Manvich DF, Stowe TA, Godfrey JR, Weinshenker D (2016): A method for psychosocial stress-induced reinstatement of cocaine seeking in rats. Biol. Psychiatry. 79(11): 940-46.

54. Oliveira VE de M, Lukas M, Wolf HN, Durante E, Lorenz A, Mayer A-L, et al. (2021): 
Oxytocin and vasopressin within the ventral and dorsal lateral septum modulate aggression in female rats. Nat. Commun. 12(1): 2900.

55. Beyeler A, Chang C-J, Silvestre M, Lévêque C, Namburi P, Wildes CP, et al. (2018): Organization of valence-encoding and projection-defined neurons in the basolateral amygdala. Cell Rep. 22(4): 905-18.

56. Vrtička P, Sander D, Vuilleumier P (2012): Lateralized interactive social content and valence processing within the human amygdala. Front. Hum. Neurosci. 6: 358.

57. Felix-Ortiz AC, Tye KM (2014): Amygdala inputs to the ventral hippocampus bidirectionally modulate social behavior. J. Neurosci. 34(2): 586-95.

58. Swanson LW, Petrovich GD (1998): What is the amygdala? Trends Neurosci. 21(8): 32331.

59. Aid T, Kazantseva A, Piirsoo M, Palm K, Timmusk T (2007): Mouse and rat bdnf gene structure and expression revisited. J. Neurosci. Res. 85(3): 525-35.

60. Maynard KR, Hill JL, Calcaterra NE, Palko ME, Kardian A, Paredes D, et al. (2016): Functional role of bdnf production from unique promoters in aggression and serotonin signaling. Neuropsychopharmacology. 41(8): 1943-55.

61. Gothard KM (2020): Multidimensional processing in the amygdala. Nat. Rev. Neurosci. 21(10): 565-75.

62. Rosenberger LA, Eisenegger C, Naef M, Terburg D, Fourie J, Stein DJ, et al. (2019): The human basolateral amygdala is indispensable for social experiential learning. Curr. Biol. 29(20): 3532-3537.e3.

63. de Gelder B, Terburg D, Morgan B, Hortensius R, Stein DJ, van Honk J (2014): The role of human basolateral amygdala in ambiguous social threat perception. Cortex. 52: 28-34.

64. Zheng J, Anderson KL, Leal SL, Shestyuk A, Gulsen G, Mnatsakanyan L, et al. (2017): Amygdala-hippocampal dynamics during salient information processing. Nat. Commun. 8: 14413. 
65. Spalletta G, Morris DW, Angelucci F, Rubino IA, Spoletini I, Bria P, et al. (2010): BDNF val66met polymorphism is associated with aggressive behavior in schizophrenia. Eur. Psychiatry. 25(6): 311-13.

66. Ernst C, Marshall CR, Shen Y, Metcalfe K, Rosenfeld J, Hodge JC, et al. (2012): Highly penetrant alterations of a critical region including bdnf in human psychopathology and obesity. Arch. Gen. Psychiatry. 69(12): 1238-46.

67. Han JC, Thurm A, Golden Williams C, Joseph LA, Zein WM, Brooks BP, et al. (2013): Association of brain-derived neurotrophic factor (bdnf) haploinsufficiency with lower adaptive behaviour and reduced cognitive functioning in wagr/11p13 deletion syndrome. Cortex. 49(10): 2700-2710.

68. Purves KL, Coleman JRI, Meier SM, Rayner C, Davis KAS, Cheesman R, et al. (2020): A major role for common genetic variation in anxiety disorders. Mol. Psychiatry. 25(12): 3292-3303.

69. van den Berg WE, Lamballais S, Kushner SA (2015): Sex-specific mechanism of social hierarchy in mice. Neuropsychopharmacology. 40(6): 1364-72.

70. Chovnick A, Yasukawa NJ, Monder H, Christian JJ (1987): Female behavior in populations of mice in the presence and absence of male hierarchy. Aggress. Behav. 13(6): 367-75.

71. Li Y, Dulac C (2018): Neural coding of sex-specific social information in the mouse brain. Curr. Opin. Neurobiol. 53: 120-30.

72. Karlsson SA, Haziri K, Hansson E, Kettunen P, Westberg L (2015): Effects of sex and gonadectomy on social investigation and social recognition in mice. BMC Neurosci. 16: 83.

73. Elsabbagh M, Divan G, Koh Y-J, Kim YS, Kauchali S, Marcín C, et al. (2012): Global prevalence of autism and other pervasive developmental disorders. Autism Res. 5(3): 160-79. 
74. Riecher-Rössler A (2017): Sex and gender differences in mental disorders. Lancet Psychiatry. 4(1): 8-9.

75. Boyd A, Van de Velde S, Vilagut G, de Graaf R, O’Neill S, Florescu S, et al. (2015): Gender differences in mental disorders and suicidality in europe: results from a large cross-sectional population-based study. J. Affect. Disord. 173: 245-54.

76. Nievergelt CM, Maihofer AX, Klengel T, Atkinson EG, Chen C-Y, Choi KW, et al. (2019): International meta-analysis of ptsd genome-wide association studies identifies sex- and ancestry-specific genetic risk loci. Nat. Commun. 10(1): 4558.

77. Seedat S, Scott KM, Angermeyer MC, Berglund P, Bromet EJ, Brugha TS, et al. (2009): Cross-national associations between gender and mental disorders in the world health organization world mental health surveys. Arch. Gen. Psychiatry. 66(7): 785-95.

78. Blokland GAM, Grove J, Chen C-Y, Cotsapas C, Tobet S, Handa R, et al. (2020): Sexdependent shared and non-shared genetic architecture across mood and psychotic disorders. BioRxiv

79. Chan CB, Ye K (2017): Sex differences in brain-derived neurotrophic factor signaling and functions. J. Neurosci. Res. 95(1-2): 328-35.

80. Puralewski R, Vasilakis G, Seney ML (2016): Sex-related factors influence expression of mood-related genes in the basolateral amygdala differentially depending on age and stress exposure. Biol. Sex Differ. 7: 50.

81. Carbone DL, Handa RJ (2013): Sex and stress hormone influences on the expression and activity of brain-derived neurotrophic factor. Neuroscience. 239: 295-303.

82. Lee G, Gammie SC (2009): GABA(a) receptor signaling in the lateral septum regulates maternal aggression in mice. Behav. Neurosci. 123(6): 1169-77.

83. Nelson RJ, Trainor BC (2007): Neural mechanisms of aggression. Nat. Rev. Neurosci. 8(7): 536-46.

84. Maynard KR, Tippani M, Takahashi Y, Phan BN, Hyde TM, Jaffe AE, et al. (2020): 
bioRxiv preprint doi: https://doi.org/10.1101/2021.1021.464669; this version posted October 22, 2021. The copyright holder for this preprint

(which was not certified by peer review) is the author/funder, who has granted bioRxiv a license to display the preprint in perpetuity. It is made available under aCC-BY-ND 4.0 International license.

Dotdotdot: an automated approach to quantify multiplex single molecule fluorescent in situ hybridization (smfish) images in complex tissues. Nucleic Acids Res.

85. Schindelin J, Arganda-Carreras I, Frise E, Kaynig V, Longair M, Pietzsch T, et al. (2012):

Fiji: an open-source platform for biological-image analysis. Nat. Methods. 9(7): 676-82. 
A
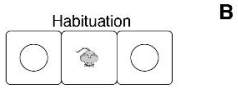

Trial 1

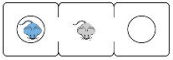

Intertrial Interval

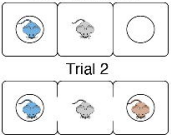

C

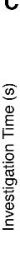

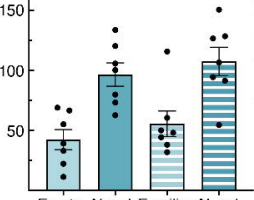

Empty Novel Familiar Novel Trial 1
Trial 2

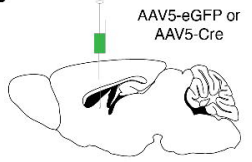

TrkB $^{\text {flf }}$ Mice

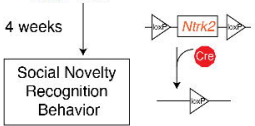

D

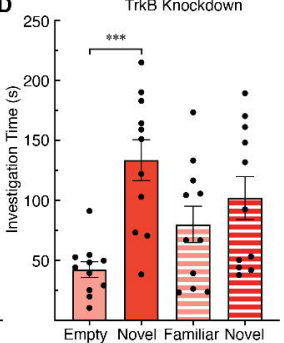

Trial 1

Trial 2

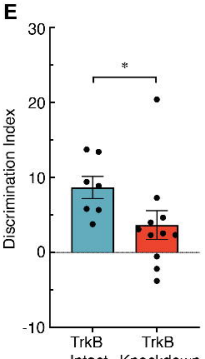

Intact Knockdown 
vCA1-LS Projection Ablation

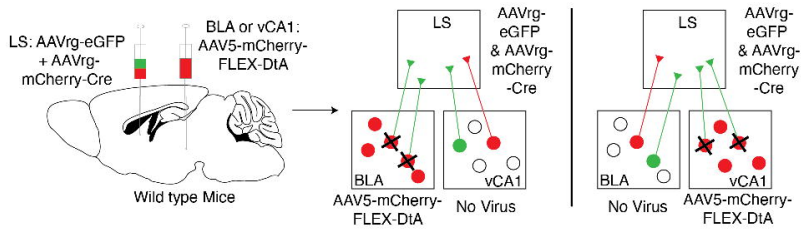

B
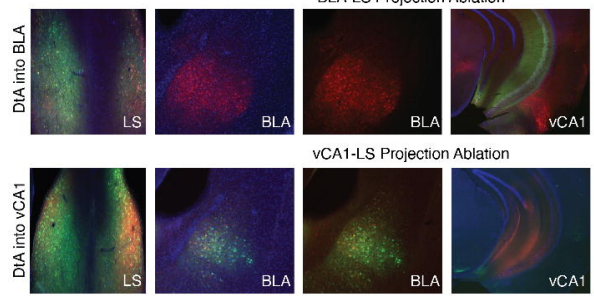

BLA-LS Projection Ablation

c

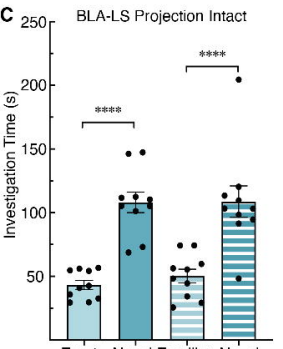

Empty Novel Familiar Novel

Trial 1

Trial 2

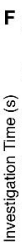

vCA1-LS Projection Intact

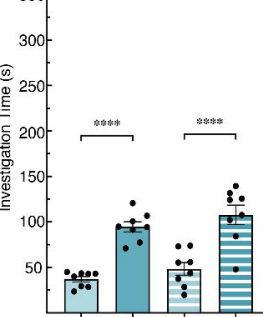

Empty Novel Familiar Novel

Trial 1

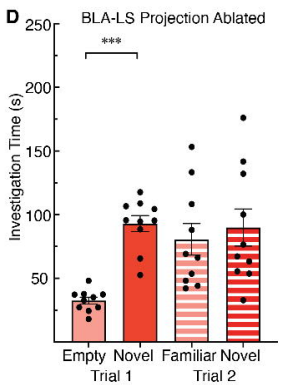

$\mathbf{G}_{350}$ vCA1-LS Projection Ablated

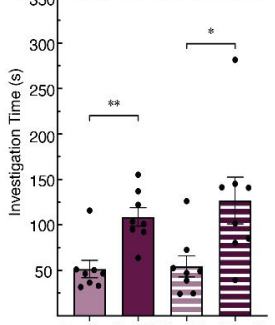

Empty Novel Familiar Novel

Trial 1

Trial 2

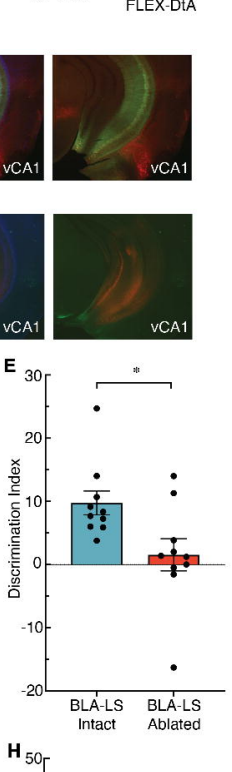

$\mathbf{H}_{50}$

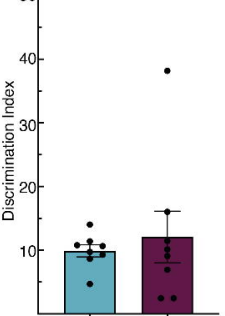

BL'A- 

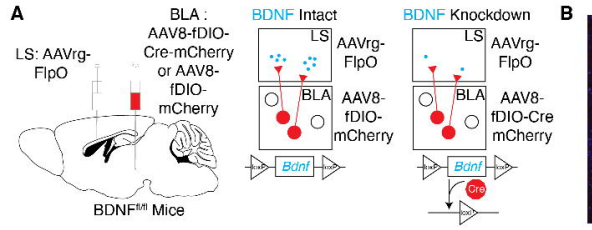

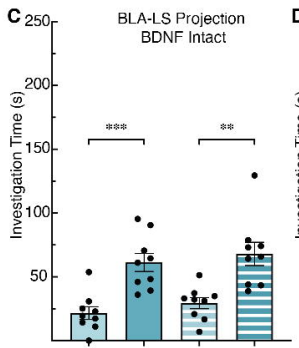

Empty Novel Familiar Novel

Trial 1

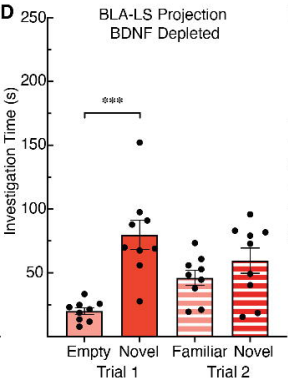

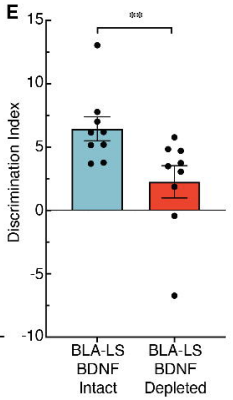

